\title{
Bioma cerrado, fragmentação florestal e relações ecológicas com a avifauna
}

\author{
Bruno Senna Corrêa, CEFET-MG-Campus IX-Nepomuceno - bruno.senna@gmail.com, \\ Júlio Neil Cassa Louzada
}

\section{RESUMO}

O Bioma Cerrado ocupa grande parte do Brasil central. Destaca-se por apresentar formações savânicas e grande diversidade de aves. O presente trabalho teve por objetivo levantar informações teóricas sobre alguns aspectos ecológicos envolvendo a fragmentação no Bioma Cerrado associada à distribuição de aves. O processo de fragmentação observado nas formações florestais do Cerrado gerou uma série de distúrbios que afetou a distribuição, estabelecimento e comportamento das aves silvestres. A fragmentação influencia diretamente na distribuição dos diversos grupos de aves. Os grupos generalistas, conseguem se deslocar pela matriz antropizada. Já os grupos dependentes de ambientes florestais, dependem de atributos mais complexos de recursos alimentares e condições especiais, para que seu deslocamento seja viabilizado. Por outro lado, a configuração da paisagem, sua estrutura espacial e os graus de conectividade, podem servir como estratégias para propiciar o deslocamento de alguns grupos, dispersores de sementes. Observa-se dessa forma a importância e relevância da presença de corredores ecológicos na conexão de fragmentos, como estratégias essenciais para manutenção do fluxo gênico, da dinâmica ecológica e da manutenção de remanescentes florestais.

Termos para indexação: Cerrado, fragmentação, ecologia de aves, diversidade de fauna

\section{Cerrado Biome, forest fragmentation and ecological relations with bird species}

\begin{abstract}
Cerrado occupies larger part of Central Brazil. This environment is distinguished for presenting savanna formations and great diversity of birds. The present work had for objective to raise theoretical information on some ecological aspects being involved the spalling in the associated Cerrado Biome to the distribution of birds. The fragmentation has generated lot of riots that affected the distribution, establishment and behavior of the wild birds. Fragmentation influences the bird distribution along the matrix. Bird forest groups demands more specific resources to cross these patches. On the other side the landscape configuration, its special structure and connectivity, could facilitate some bird groups distribution, as dispersive bird families. It is necessary to better understand, the importance of ecological corridors in the connection of deforested areas, as essential strategies for maintenance of the genic flow, the ecological dynamics and the maintenance of forest fragments species diversity.
\end{abstract}

Indexed expressions: process of spalling, bird ecology, fauna diversity 


\section{INTRODUÇÃO}

O Cerrado é um bioma que apresenta área superior a dois milhões de quilômetros quadrados. Trata-se de uma vegetação com fitofisionomias que englobam formações florestais, savânicas e campestres, condicionadas pelo clima, latitude, química e física do solo, disponibilidade de água e fatores antrópicos (Barbosa \& Schmiz, 1998). Os distúrbios provocados no Bioma formam matrizes vegetacionais como uma colcha de retalhos, marcando limites que podem ser considerados barreiras geográficas. Associada a essas matrizes, observa-se a expansão agrícola e urbana que vêm alterando as fitofisionomias naturais. A legislação ambiental, na tentativa de estabelecer critérios e regras para conter a expansão agrícola de forma ordenada e controlada, estabeleceu o Código Florestal Brasileiro (Lei $\mathrm{n}^{\circ} 4.771$, de 15 de setembro de 1965) - (D.O.U. de 16/09/65).

Entre os fatores que alteram as fitofisionomias naturais e a distribuição e dispersão das comunidades avifaunísticas, observa-se a fragmentação florestal. A fragmentação gera alterações nos habitats naturais, modificando os nichos e o microclima dos ambientes (fatores abióticos e fatores bióticos), reduzindo assim a disponibilidade de recursos alimentares (qualidade e/ou quantidade), espaço e abrigo (Turner, 1996).

\section{REFERENCIAL TEÓRICO}

A avifauna do Cerrado engloba diversas espécies residentes e migratórias, que estabelecem comportamentos específicos ao longo das estações do ano. Tais comportamentos como o tipo de forrageamento e a procura de espaço para abrigo e nidificação, permitem observar diferentes grupos de aves ao longo do ano: insetívoros, na primavera e verão; granívoros e frugívoros com distribuição sazonal; onívoros durante todo o ano
(Sick, 2001). A distribuição das comunidades de aves do Cerrado, também está relacionada aos gradientes topográfico e vegetacional. Observam-se grupos de aves generalistas que apresentam ampla distribuição e dieta diversificada, e grupos especialistas, dependentes de ambientes não antropizados, como as espécies florestais, de dieta e comportamento específicos (Sick, 2001).

\subsection{Bioma Cerrado}

O Cerrado é considerado um complexo vegetacional com características ecológicas e fisionômicas similares a outras savanas da América tropical, da África e da Austrália (Eiten, 1994). Por se tratar do bioma que mais sofre pressão humana no Brasil (Ratter et al., 1997), seja pelas queimadas (Ribeiro \& Silva, 1996), ou pela expansão agrícola, observa-se que a cobertura original do cerrado brasileiro já foi reduzida em mais de 37\% (Felfili, 2003). Essa redução de áreas, associada ao isolamento altera a estrutura do ambiente comprometendo sua biodiversidade, aumentando o risco de extinções (Metzger, 1999).

A riqueza de espécies e a grande variabilidade de sua distribuição nas diferentes fitofisionomias dependem basicamente de fatores naturais e antrópicos (Ribeiro \& Silva, 1996). Entre os fatores naturais que mantêm a biodiversidade, encontram-se aqueles que atuam nos processos de formação do bioma como clima, topografia e características químicas do solo. Quanto aos fatores antrópicos que, em geral, desfavorecem a biodiversidade, destacam-se o extrativismo e as atividades agrícolas.

Segundo Mendonça et al. (1998) o Bioma apresenta-se como uma das mais ricas savanas do mundo, cuja flora é composta por 6.420 espécies vasculares. O cerrado sensu stricto, uma das fitofisionomias mais representativas do Bioma ( $70 \%$ do total), tem sua paisagem composta por um estrato herbáceo 
dominado principalmente por gramíneas, e um estrato de árvores e arbustos tortuosos, com ramificações irregulares e retorcidas, variando em cobertura de 10 a 60 \% (Eiten, 1994).

A diversidade das formações florestais associadas aos cursos de água do Cerrado, quando comparadas às de outros biomas (da Floresta Amazônica até a Floresta Atlântica), pode ser observada numa rota Noroeste-Sudoeste, através de uma rede dendrítica de florestas ciliares que cruzam o Brasil dentro do Bioma Cerrado. Essa rota fornece informações sobre os padrões florísticos ligados com a Floresta Amazônica (florestas do norte e oeste do Cerrado) ou com as florestas semidecíduas montanas do Sudeste brasileiro (florestas do centro e do sul) (Ratter et al., 1973; Rizzini, 1979).

\subsection{Avifauna de Cerrado}

A diversidade de aves do Cerrado engloba cerca de 856 espécies, distribuídas em 64 famílias, incluindo residentes, migrantes altitudinais e migrantes dos hemisférios Norte e Sul (CBRO, 2007). O Cerrado apresenta 36 espécies endêmicas (Silva, 1995; Cavalcanti, 1999; Silva \& Bates, 2002; Lopes, 2004), um total de 48 táxons ameaçados (Ibama, 2003; IUCN, 2007) 14 táxons endêmicos do Bioma e 14 táxons endêmicos do Brasil (Ibama, 2003; IUCN, 2007). A sua distribuição, entre outros fatores está associada à grande variedade de frutos, ocorrência de abrigos naturais e clima (Barbosa \& Schmiz, 1998). Para o estado de Minas Gerais, segundo Collar et al. (1994), das 780 espécies presentes, 83 fazem parte da lista de espécies ameaçadas do Estado - Deliberação COPAM 041/95.

Trabalhos relacionando a avifauna do Cerrado envolvem parâmetros como os tipos de ambientes (Braz \& Cavalcanti, 2001; Martins, 2001; Fanchin, 2004; Valadão et al., 2006), endemismos (Silva, 1997; Silveira \& D’Horta, 2001), estudos de conservação (Sil- va, 1998a; Cavalcanti, 1999; Straube et al., 2005; Correa et al., 2007), a riqueza de espécies (Urben-Filho et al., 2000; Braz et al., 2002; Fontes et al., 2002), dieta (Mercival \& Galetti, 2002; Manhães, 2003; Siqueira et al., 2007), bandos mistos (Silva, 1980; Alves, 1988), reprodução (Macedo, 1992; Marini \& Cavalcanti, 1992; Marini et al., 2006), comportamento (Cavalcanti \& Pimentel, 1988; Alves, 1990; Marini, 1992; Barbosa, 1999; Ragusa-Neto, 2000; Marcondes-Machado, 2002), biogeografia (Silva, 1998b; Cavalcanti, 1990; Nunes \& Tom, 2004) entre outros.

\subsection{Fragmentação do Cerrado e avifauna}

O processo de expansão agrícola nas últimas décadas tem provocado um aumento na fragmentação de florestas nativas. Tais processos provocam distúrbios sobre o ambiente, seja na modificação de elementos da estrutura física dos ecossistemas, seja na estrutura da paisagem como um todo (Valério Filho, 1995). Viana (1990) sugere o termo fragmento como uma área interrompida por barreiras antrópicas (estradas, pastagens) ou naturais (montanhas, lagos) com capacidade de reduzir o fluxo de animais, pólen ou sementes. Nesse contexto, Cerqueira et al. (2003), definiram fragmentação como o processo no qual um habitat contínuo é dividido em manchas, ou fragmentos, mais ou menos isolados.

A fragmentação é um processo de mudanças na escala espacial importante na evolução e conservação. Tal processo promove a emergência de descontinuidades em ambientes de paisagem (Groom \& Grubb, 2006). Pode ser causada por processos geológicos que, lentamente, alteram a camada do ambiente físico através de atividades humanas como conversão da terra, para agricultura e pecuária. Esses dois últimos processos alteram o ambiente mais rapidamente, em escala de tempo. Entre os processos supracitados, os primeiros parecem estar relacionados como 
as principais causas da especiação das espécies. O segundo (atividades humanas) parecem estar mais relacionados a promover a extinção de muitas espécies. A fragmentação é freqüentemente causada pelo homem, quando promove destruição de vegetação nativa para atividades como agricultura, desenvolvimento rural ou urbanização. Os habitats outrora contínuos, passam a se tornar divididos em fragmentos separados. Após desmatamento intenso, os fragmentos separados tendem a se tornar pequenas ilhas isoladas cada qual por culturas agrícolas, pastagens, terras estéreis, etc. (Noss et al., 2006; Wickam et al., 2007). O termo fragmentação de habitats inclui, pelo menos, seis fenômenos: redução da área total do habitat; crescimento da quantidade de borda; decréscimo de habitat de interior; isolamento de um habitat do fragmento para outras áreas de habitat; quebra de um pedaço de habitat em vários pedaços menores; decréscimo do tamanho médio de cada pedaço de habitat (Rosenzweig, 1995).

O processo de fragmentação apresenta alguns aspectos importantes como a perda do habitat original, a redução do tamanho do remanescente, o aumento de isolamento de remanescentes (Andrén, 1994) e a exposição do fragmento florestal ao efeito borda, como resultado da transição abrupta entre floresta e matriz (Murcia, 1995). Com a fragmentação florestal, é inevitável a criação de bordas artificiais que podem implementar transformações aos sistemas biológicos, ocasionadas em grande parte por efeitos de borda (Rodrigues $\&$ Nascimento, 2006).

Entre outros fatores decorrentes desse processo observam-se, ainda, variações na intensidade de luz e de recursos alimentares (Murcia, 1995). O tamanho do fragmento e a densidade populacional das espécies de aves florestais serão diretamente influenciados por essas variações.

Entre os representantes de fauna silvestre em ambientes fragmentados, os maio- res dispersores são as aves. Entre os critérios para avaliação dos usuários potenciais nos corredores, observam-se: 1) o status de distribuição das espécies; 2) o grau de dependência de habitats florestais; 3 ) o grau de mobilidade através de áreas abertas; 4) o grau de especialização de habitat; 5) a resposta das espécies ao desmatamento (sensível, não-sensível); 6) o status de conservação das espécies. Entretanto, deve-se levar em consideração que existe uma relação direta entre fauna e flora e esse fator é fundamental para o sucesso reprodutivo das espécies e manutenção do equilíbrio dinâmico desses ambientes. Poucos trabalhos relacionam a comunidade de aves em ambientes de transição do Cerrado e outras fitofisionomias (Antunes, 2005).

Existem pelo menos dois aspectos fundamentais para discutir o efeito da fragmentação sobre as aves: a) o comportamento das aves; b) a configuração da paisagem fragmentada (fragmentos, matriz, corredores) (Sttoufer \& Bierregaard, 1995b).

\section{a) Comportamento das aves}

Os animais frugívoros exercem um importante papel na demografia das comunidades vegetais, pois: a) interagem no momento em que as plantas estão no estágio final do ciclo reprodutivo, podendo favorecer ou comprometer o sucesso dessa fase (Jordano, 1989); b) o padrão de deposição das sementes, no ambiente, pelos frugívoros afeta diretamente a sobrevivência das sementes e o estabelecimento das plântulas (Howe, 1986; Katusic-Malmborg \& Willson, 1988), e c) promovem a distribuição espacial dos futuros indivíduos adultos na floresta ao descartar as sementes após a ingestão (Jordano, 1992). Essas considerações são relevantes uma vez que, nas florestas tropicais, 50 a 90\% das espécies arbóreas produzem frutos adaptados para a dispersão por animais (Howe \& Smallwood, 1982). 
Em relação ao comportamento das aves, podem-se relacionar os hábitos alimentares, com diferentes estratégias de forrageamento e os processos de nidificação diretamente relacionados à disponibilidade de recursos alimentares, para permanência em determinada área. Esse último parâmetro está diretamente relacionado com a distribuição da estrutura populacional de espécies vegetais de dispersão zoocórica. A estratégia reprodutiva é um dos principais fatores que levam as aves a buscar recursos energéticos para poder cumprir seu ciclo biológico. Para obter energia necessária aos processos vitais e à perpetuação da espécie, o processo evolutivo das aves permitiu o desenvolvimento de características específicas que incluem memória de localização, visão desenvolvida e estratégias complexas para forrageamento de recursos alimentares (Aleixo \& Vielliard, 1995). A fragmentação, a redução das áreas e as bordas resultantes podem alterar a abundância de plantas frutíferas e aves frugívoras (Restrepo et al., 1999). Conseqüentemente, mudanças na composição das espécies de aves em fragmentos pequenos podem afetar a probabilidade de dispersão de sementes, podendo alterar a comunidade de sub-bosque e, assim a dinâmica vegetal do fragmento ( $\mathrm{Ga}$ letti et al., 2003).

\section{b) Conformação da paisagem fragmentada e isolamento}

Os principais parâmetros relacionados com a paisagem fragmentada envolvem o tamanho e a forma do elemento da paisagem (Saunders \& Hobbs, 1991). Fragmentos mais alongados tendem a sofrer efeitos de borda mais intensos (Laurance \& Bierregaard Junior, 1997). Os efeitos de fragmentação do habitat são controlados por dois processos principais: os efeitos internos nos fragmentos ligados à formação da borda de floresta e a influência externa do habitat matriz, na dinâmica do fragmento (Gascon et al., 1999). A influência externa da matriz inclui interação da paisagem em aspecto mais amplo de configuração de habitat (porções de matriz e conectividade) (Fahring \& Merrian, 1994).

A configuração de uma paisagem está associada à distribuição física dos elementos que a compõem, os parâmetros relacionados com a estrutura espacial e os graus de conectividade (McGarigal \& Marks, 1995). Essa conectividade é um evento que depende da presença de corredores ou algum tipo de ligação, similar ao habitat primário, visando permitir a movimentação e dispersão entre fragmentos (Harrison, 1992).

Os habitats alterados na matriz circunvizinha de paisagem são uma fonte de colonizadores potenciais (Stouffer \& Bierregaard Junior, 1995a,b; Laurance \& Bierregaard Junior, 1997; Laurance et al., 1997; Renjifo, 2001). Em relação à ocorrência desse tipo de paisagem, para fragmentos florestais tropicais, observa-se maior atenção aos valores de conservação de terras fora de reservas que incluem ambientes fragmentados, florestas secundárias, terras particulares e habitats de campo como plantações de café (Brown \& Lugo, 1990; Turner \& Corlett, 1996; Daily, 2001).

O grau de isolamento do fragmento, isto é, uma medida de quão separado o fragmento está de outras áreas que podem servir como fonte de animais e sementes, é um fator importante para as interações entre as plantas e os dispersores de sementes (Estrada et al., 1993). Muitos animais frugívoros não atravessam áreas abertas ou evitam ambientes perturbados (Estrada et al., 1993; Silva et al., 1996).

Dessa forma, fragmentos isolados tendem a receber menos sementes de outras áreas e apresentar menor abundância e riqueza de animais frugívoros. A literatura mostra que o grau de isolamento de um fragmento está negativamente relacionado com a ri- 
queza de plantas zoocóricas (Ruremonde \& Kalkhoven, 1991; Ochoa-Gaona et al., 2004). Os efeitos do isolamento podem ser minimizados pela composição da matriz que circunda o fragmento e, em maior escala, pela composição da paisagem onde o fragmento está inserido (Metzger, 2000). Esses dois fatores podem influenciar a conectividade do ambiente e, portanto, o fluxo de animais e sementes no fragmento (Metzger, 2000; Graham, 2001).

Santos (2004), estudando a comunidade de aves em ambientes fragmentados de vegetação secundária, observou correlação positiva entre o número de espécies e o tamanho das áreas. Entretanto foi observada similaridade entre a distribuição da riqueza associada a ambientes perturbados, mas a riqueza apresentou correlação negativa com o tamanho do fragmento. Antogiovanni \& Metzger (2005) destacaram a influência da matriz e do tamanho de fragmentos florestais pequenos na Amazônia, no número de espécies de aves insetívoras. Em trabalhos dessa natureza, a redução de habitat dificulta o estabelecimento de grupos de interior, sensíveis a alterações e que apresentam hábitos específicos (Stouffer \& Bierregaard Junior, 1995b; Stratford \& Stouffer, 2001; Ferraz et al., 2003).

Uezu et al. (2008), buscando relacionar a distribuição de aves em remanescentes florestais funcionando como stepping stones (Fig. 1) em área de Floresta Atlântica, observaram o predomínio de espécies generalistas e de áreas abertas em detrimento de espécies florestais. Fatores como as distâncias entre fragmentos foram essenciais para determinar a riqueza e abundância de espécies florestais. A literatura confirma existir uma relação entre a permeabilidade da matriz e a eficiência de stepping stones, que geralmente funciona em graus intermediários de resistência da matriz (Uezu, 2002, 2006; Uezu et al., 2005).

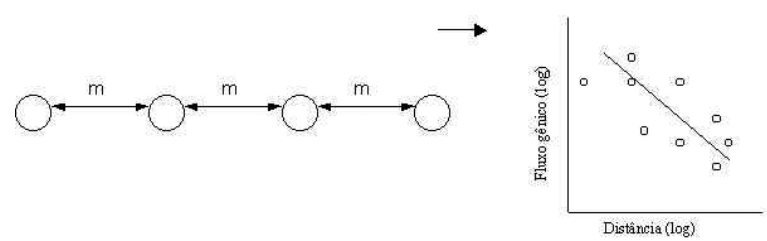

Figura 1: Modelo de "stepping stone" (Fonte: modificada de Vrjenhoek 1997)

Estudos envolvendo a permeabilidade da matriz vêm sendo realizados no intuito de esclarecer os parâmetros e se há padrões que podem facilitar o seu funcionamento e se existem relações entre o grau de conectividade das paisagens com modelos de metapopulações (Burgess, 1988; Fahrig \& Merrian, 1994; Metzger, 1997; Wiens et al., 1997; Hanski, 1998; Moilanen \& Hanski, 1998).

Assim, se entendemos que a biodiversidade vai além do conjunto de espécies que compõem o ambiente e engloba também as interações entre elas, justifica-se plenamente todo o esforço de manejo dos fragmentos e da paisagem circundante, para minimizar os efeitos da fragmentação sobre as interações entre as plantas e os dispersores de suas sementes (Metzger, 2000).

\subsection{Corredores ecológicos}

Os corredores ecológicos representam uma das estratégias mais promissoras para o planejamento regional eficaz de conservação e preservação de flora e fauna de áreas fragmentadas. A ligação desses remanescentes isolados por corredores de vegetação natural é uma estratégia para mitigar os efeitos da ação antrópica e garantir a biodiversidade nos mesmos (Valeri \& Senô, 2004).

$\mathrm{Na}$ tentativa de se conservar fragmentos remanescentes do Cerrado, pode-se observar a presença de corredores de fauna. Corredores ecológicos ou corredores de fauna compreendem faixas de vegetação que podem conectar dois ou mais fragmentos florestais ou sair de um fragmento e terminar 
em um ponto cego (Boone \& Hunter 1996; Saunders \& Hobbs, 1991; Metzger, 1997). O estudo de corredores ecológicos tem sido realizado há, pelo menos, 50 anos na Austrália e nos Estados Unidos, países com paisagens já bastante fragmentadas (Saunders \& Hobbs, 1991). Na América do Sul, trata-se de uma proposta que vem sendo aplicada em projetos relacionados à Floresta Atlântica e Floresta Amazônica e tem despertado contínuo interesse no processo de conservação e manutenção da biodiversidade (Laurance \& Bierregaard, 1997).

Os corredores de vegetação podem ser definidos como um elemento de paisagem linear bidimensional que conecta dois ou mais fragmentos de vegetação; funciona basicamente como conduto para animais silvestres (Soulé \& Gilpin, 1991). A conectividade ou conexão se refere ao grau, no qual o isolamento absoluto é impedido pelos elementos da paisagem, permitindo aos organismos se moverem entre fragmentos (Merriam, 1984).

Os processos ecológicos que delimitam a importância demográfica dos corredores dependem das respostas comportamentais dos animais, em termos de movimentação (Saunders \& Hobbs, 1991; Merriam, 1991). Uma espécie pode necessitar de conexões entre os fragmentos, por razões não relacionadas aos processos demográficos de uma metapopulação, mas sim por fatores comportamentais ou espaciais (Merriam, 1991). A conectividade entre fragmentos deve ser alta o suficiente para assegurar baixos riscos na movimentação da fauna, pois o uso contínuo dos recursos pode gerar esgotamento dos mesmos (Merriam, 1991).

O tipo de movimento pelos corredores pode ser classificado em três categorias: (1) caminhamento aleatório; (2) dependente de densidade; e (3) direcional, incluindo deslocamento entre áreas de forrageamento sazonal e áreas de reprodução e nascimentos (Merriam, 1991). A periodicidade da movi- mentação de fauna é geralmente marcada por mudanças ambientais ou ciclos (diurno, lunar ou sazonal) ou ainda pela história de vida. Dessa forma, são objetivos dos corredores, entre outros, facilitar a migração para reprodução ou sítios para hibernação e o forrageamento entre fragmentos (Soulé \& Gilpin, 1991; Thorne, 1993).

Entre outros fatores relacionados com a capacidade dos corredores, destacam-se: efeito borda, tipo de habitat, qualidade e diversidade de habitat, dimensão, largura, forma, história de vida, tipo de movimentação, tempo e periodicidade, efeitos de interações intra-específicas/interespecíficas (Saunders \& Hobbs, 1991). As bordas dos corredores podem apresentar características físicas e biológicas distintas, como a presença de espécies vegetais frutíferas atrativas às aves, estrutura vertical favorável (presença de poleiros, galhos, árvores altas e de porte médio, densas ou pouco adensadas), ambiente sombrio e úmido. A presença de espécies vegetais polinizadas por insetos, na borda, podem fornecer disponibilidade de recursos a aves insetívoras, favorecendo o deslocamento dessas espécies pelos corredores (Saunders \& Hobbs, 1991).

A conectividade entre fragmentos pode ser avaliada de duas maneiras: uso de corredores somente como rota de fauna, independente de recursos; uso de corredores como rota de fauna, dependente de recursos. No primeiro caso, avalia-se somente a taxa de mortalidade. No segundo caso, observa-se a existência de variáveis espaciais, temporais e comportamentais. Esses dois casos ilustram outros dois possíveis mecanismos de movimentação entre fragmentos: a movimentação de fauna pode provocar o deslocamento de indivíduos do fragmento A para o corredor e deslocamento do corredor para o fragmento B; indivíduos podem se deslocar, por exemplo entre dois fragmentos conectados, através do corredor sem se tornarem residentes nos corredores (Merriam, 1984). 
Fatores como a forma, a largura, a composição de espécies vegetais, a distância, a influência da matriz circunvizinha e as conseqüências dessa matriz, na estrutura dos corredores, são essenciais para a avaliação dos processos ecológicos. A forma dos corredores artificiais criados visa o deslocamento de fauna. Tais ambientes são geralmente planejados através de fotografias aéreas e tendem a ser lineares. Esse formato facilita o deslocamento de répteis, aves e mamíferos. A largura deve ser mensurada em conjunto com outros fatores como composição de espécies vegetais e matriz circunvizinha. Corredores muito largos $(50 \mathrm{~m}-100 \mathrm{~m})$ podem sofrer efeitos de fatores como fogo, construção de estradas, efeito-borda, gerando alterações na dinâmica da comunidade do corredor e dos fragmentos conectados, declinando assim sua eficiência, ao longo dos anos (Saunders \& Hobbs, 1991; Saunders et al., 1991).

Devido às evidências de que corredores e fragmentos aumentam a sustentabilidade do ambiente, alternativas de manejo de corredores são sugeridas. A implantação de faixas de árvores ao longo de estradas e cercas, o aumento da largura e o estabelecimento de matas ripárias (conforme indicado pela legislação), podem ser usados como formas de promover os avanços na conservação da biodiversidade das espécies (Harris, 1984; Forman, 1997).

\section{CONCLUSÃO}

A fragmentação limita o deslocamento de espécies florestais, reduz a população de grupos especialistas e contribui para a redução do fluxo gênico de grupos animais e vegetais. Observa-se como fator ideal a disponibilização de fragmentos florestais acima de 80 ha, para fins de conservação de populações relictuais de aves. Entretanto como a realidade do Cerrado está ligada historicamente a um processo gradual de supressão de vegetação nativa em decorrência do avanço das fronteiras agrícolas, isso não é possível. Entre as alternativas sugeridas para a manutenção da diversidade de fauna, especialmente para aves, está ligado a fiscalização ambiental (política conservacionista) e a execução de Planos de Manejo e Monitoramento associado a culturas agrícolas representativas do Bioma em questão (café por exemplo), desde que englobem conexão de remanescentes florestais e proteção de fragmentos nativos de fatores como: fogo, gado, retirada de lenha, desmatamentos e substituição de vegetação nativa por eucalipto.

\section{REFERÊNCIAS BIBLIGRÁFICAS}

ALEIXO, A.; VIELliARD, J. M. E. Composição e dinâmica da avifauna da mata de Santa Genebra, Campinas, São Paulo, Brasil. Revista Brasileira de Zoologia, Viçosa, v. 12, n. 3, p. 493-511, 1995.

\section{ALVES, M. A. S. Organização social e bio- logia reprodutiva de Neothraupis fasciata (Passeriformes: Thraupidae). 1988. Dis- sertação (Mestrado) - Universidade Estadual de Campinas, Campinas.}

ALVES, M. A. S. Social system and helping behavior in the white-banded tanager (Neothraupis Fasciata). Condor, Santa Clara, v. 92, n. 2, p. 470-474, Mar./Apr. 1990.

ANDRÉN, H. Effects of habitat fragmentation on birds and mammals in landscapes with different proportions of suitable habitat: a review. Oikos, Lund, v. 71, n. 1, p. 355-366, 1994.

ANTOGIOVANNI, M.; METZGER, J. P. Influence of the matrix habitats on the occurrence of insectivorous bird species in Amazonian forest fragments. Biological Conservation, Kidlington, v. 122, n. 3, p. 441-451, 2005. 
ANTUNES, A. Z. Alterações na composição da comunidade de aves ao longo do tempo em um fragmento florestal no sudeste do Brasil. Revista Brasileira de Ornitologia, Ararajuba, v. 13, n. 1, p. 47-61, Jun. 2005.

\section{BARBOSA, A. A. A. Hortia brasiliana} Vand. (Rutaceae): polinização por aves Passeriformes no cerrado do sudeste brasileiro. Revista Brasileira de Botânica, São Paulo, v 22, n. 1, p. 99-105, 1999. Disponível em: <http://www.scielo.br/ scielo.php? script $=$ sci_arttext\&pid $=\mathrm{S} 0100$ $-84041999000100013 \& \operatorname{lng}=\mathrm{pt} \& \mathrm{nrm}=$ iso $>$. Acesso em: 1 dez. 2007.

BARBOSA, A. S.; SCHMIZ, P. I. Ocupação indígena do cerrado: esboço de uma história. In: SANO, S. M.; ALMEIDA, S. P. Cerrado: ambiente e flora. Planaltina: EmbrapaCPAC, 1998. p. 3-42, 556 p.

BOONE, R. B.; HUNTER, M. L. Using diffusion models to simulate the effects of land use on grizzly bear dispersal in the Rocky Mountains. Landscape Ecology, Amsterdam, v. 11, n. 1, p. 51-64, 1996.

BRASIL. Congresso. Senado. Legislativo. Ministério do Meio Ambiente-MMA. Lei $\mathrm{n}^{\mathrm{o}} 4.771$ de 15 de setembro de 1965. Institui o novo Código Florestal. Diário Oficial [da] República Federativa do Brasil, Poder Executivo, Brasília, DF, 15 set. 1965, p. 9529, setembro de $1965.17 \mathrm{p}$.

BRAZ, V. S.; ABREU, T.; LOPES, L. E.; LEITE, L. O.; FRANÇA, F. G. R. Levantamento preliminar da avifauna da região do Jalapão, Tocantins. In: CONGRESSO BRASILEIRO DE ORNITOLOGIA, 10., 2002, Fortaleza. Resumos... Fortaleza: UFCE, 2002.
BRAZ, V. S.; CAVALCANTI, R. B. A representatividade de áreas protegidas do distrito federal na conservação da avifauna do Cerrado. Revista Brasileira de Ornitologia, Ararajuba, v. 9, n. 1, p. 61-69, jun. 2001.

BROWN, S.; LUGO, A. E. Tropical secondary forests. Journal of Tropical Ecology, New York, v. 6, n. 1, p. 1-32, 1990.

BURGESS, R. L. Community organization: effects of landscape fragmentation. Canadian Journal of Botany, Ottawa, v. 66, n. 12, p. 2687-2690, Nov. 1988.

CAVALCANTI, R. B. Bird species richness and conservation in the cerrado region of central Brazil. Studies in Avian Biology, [S.1.], v. 19, n. 1, p. 244-249, 1999.

CAVALCANTI, R. B. Migrações de aves no cerrado. In: ENCONTRO NACIONAL DE ANILHADORES DE AVES, 4., 1990, Recife, PE. Anais... Recife: UFRP, 1990. p. 110-116.

CAVAlCANTI, R. B.; PIMENTEL, T. M. Shiny cowbird parasitism in central Brazil. Condor, Santa Clara, v. 90, n. 1, p. 40-43, Jan./Feb. 1988.

CERQUEIRA, R.; BRANT, A.; NASCIMENTO, M. T.; PARDINI, R. Fragmentação: alguns conceitos. In: RAMBALDI, D. M.; OLIVEIRA, D. A. S. (Orgs.). Fragmentação de ecossistemas: causas, efeitos sobre a biodiversidade e recomendações de políticas públicas. Brasília, DF: MMA, 2003. p. 23-40.

Código Florestal Brasileiro (Lei $\mathrm{n}^{\mathrm{o}}$ 4.771, de 15 de setembro de 1965) - (D.O.U. de 16/09/65). 
COLLAR, N. J.; CROSBY, M. J.; STATTERSFIELD, A. J. Birds to watch 2: the world list of threatened birds. Cambridge: Birdlife International, 1994. (Conservation Series, 4).

COMITÊ BRASILEIRO DE REGISTROS ORNITOLÓGICOS. Listas das aves do Brasil. Versão 16.8/2007. Disponível em: <http:// www.cbro.org.br>. Acesso em: 20 jan. 2007.

CORREA, J.; LEITE, L. O.; GARCIA, F. I.; MARINI, M. Â. Modelagem de nicho ecológico (GARP) para aves endêmicas do Cerrado: uma análise crítica. In: DINIZ, I.; MARINHO-FILHO, J. S.; CAVALCANTI, R. B.; MACHADO, R. B. (Orgs.). Análise da biodiversidade do Cerrado. Brasília, DF: UnB, 2007. v. 1, p. 1-30.

DAILY, G. C. Ecological forecasts. Nature, London, v. 411, n. 245, May 2001.

Deliberação COPAM 041/95.

EITEN, G. Vegetação do Cerrado. In: PINTO, M. N. (Org.). Cerrado: caracterização, ocupação e perspectivas. Brasília, DF: UnB/ SEMATEC, 1994. p. 17-73.

ESTRADA, A.; COATES-ESTRADA, R.; MERRIT JUNIOR, D. A.; MONTIEL, S.; CURIEL, D. Patterns of frugivore species richness and abundance in forest islands and in agricultural habitats at Los Tuxtlas, Mexico. Plant Ecology, Dordrecht, v. 107/108, n. 1, p. 245-257, Jun 1993.

FAHRIG, L.; MERRIAN, G. Conservation of fragmented populations. Conservation Biology, Cambridge, v. 8, n. 1, p. 50-59, Mar. 1994.

FANCHIN, A. G. A riqueza da avifauna no Parque Municipal do Sabiá, zona urbana de Uberlândia (MG). Biotemas, [S.1.], v. 17, n. 1, p. 179-202, 2004.
FELFILI, J. M. Fragmentos florestais estacionais do Brasil central: diagnóstico e proposta de corredores ecológicos. In: COSTA, R. B. Fragmentação florestal e alternativas de desenvolvimento rural na região Centro-Oeste. Campo Grande: UCDB, 2003. p. 139-160.

FERRAZ, G.; RUSSEL, J.; STOUFFER, P. C.; BIERREGAARD, R. O.; PIMM, S. L.; LOVEJOY, T. E. Rates of species loss from Amazonian forest fragments. PNAS, [S.1.], v. 100, n. 24, p. 14069-14073, Nov. 2003.

FONTES, J.; CINTRA, R.; SANAIOTTI, T. Efeito da variação na composição da comunidade de árvores na riqueza e abundância de aves em um cerrado mato-grossense. 2002. Disponível em: <http://www.cpap.embrapa.br/agencia/congresso/Bioticos/FONTES-076.pdf>. Acesso em: 30 nov. 2007.

FORMAN, R. T. T. Land mosaics: the ecology of landscapes and regions. Cambridge: University of Cambridge, 1997. 605 p.

GALETTI, M.; ALVES-COSTA, C. P.; CAZETTA, E. Effects of forest fragmentation, anthropogenic edges and fruit color on the consumption of ornithocoric fruits. Biological Conservation, Oxford, v. 111, n. 2, p. 269-273, June 2003.

GASCON, C.; LOVEJOY, T. E.; BIERREGAARD JUNIOR, R. O.; MALCOM, J. R.; STOUFFER, P. C.; VASCONCELOS, H. L.; LAURANCE, W. F.; ZIMMERMAN, B.; TOCHER, M.; BORGES, S. Matrix habitat and species richness in tropical forest remnants. Biological Conservation, Oxford, v. 91, n. 2-3, p. 223-229, Dec. 1999.

GRAHAM, C. H. Factors influencing movement patterns of Keel-billed toucans in a fragmented tropical landscape in southern Mexico. Conservation Biology, Cambridge, v. 15, n. 6, p. 1789-1798, Dec. 2001. 
GROOM, J. D.; GRUBB, T. C. Patch colonization dynamics in Carolina Chickadees ( $\mathrm{Po}$ ecile carolinensis) in a fragmented landscape: a manipulative study. Auk, [S.1.], v. 123, n. 4, p. 1149-1160, 2006.

HANSKI, I. Metapopulation dynamics. Nature, [S.1.], v. 396, p. 41-49, 1998.

HARRIS, L. D. The fragmented forest: the island biogeography theory and the preservation of biotic diversity. Chicago: University of Chicago, 1984. 211 p.

HARRISON, R. L. Toward a theory of inter-refuge corridor design. Conservation Biology, Cambridge, v. 6, n. 2, p. 293-295, Jun. 1992.

HOWE, H. F. Seed dispersal by fruit-eating birds and mammals. In: MURRAY, D. R. (Ed.). Seed dispersal. Sydney: Academic Pr. Australia, 1986. p. 123-189.

HOWE, H. F.; SMALLWOOD, J. Ecology of seed dispersal. Annual Review of Ecology and Systematics, Palo Alto, v. 13, p. 201228, Nov. 1982.

INSTITUTO BRASILEIRO DO MEIO AMBIENTE E DOS RECURSOS NATURAIS RENOVÁVEIS. Instrução Normativa n. 3, de 27 de maio de 2003. Lista das espécies da fauna ameaçada de extinção. Brasília, DF: Ministério do Meio Ambiente, 2003.

IUCN. 2007 IUCN red list of threatened species. Disponível em: <http://www.redlist. org >. Acesso em: 10 nov. 2007.

JORDANO, P. Fruits and frugivory. In: FENNER, M. (Ed.). Seeds: the ecology of regeneration in plant communities. Washington, DC: C.A.B. International, 1992. p. 105-156.
JORDANO, P. Pre-dispersal biology of Pistacia lentiscus (Anacardiaceae): cumulative effects on seed removal by birds. Oikos, Lund, v. 55, n. 3. p. 375-386, Jul. 1989.

KATUSIC-MALMBORG, P.; WILLSON, M. F. Foraging ecology of avian frugivores and some consequences for seed dispersal in an Illinois woodlot. Condor, Santa Clara, v. 90, n. 1, p. 173-186, Feb. 1988.

KIMURA, M. "Stepping stone" model of population. Ann. Rept. Nat. Inst. Genetics, v. 3, p. 62-63. 1953.

KIMURA, M. The Stepping stone model of population structure and the decrease of genetic correlation with distance. Genetics, v. 49, p. 561-576. 1964.

LAURANCE, W. F.; BIERREGAARD JUNIOR, R. O. Tropical forest remnants: ecology, management, and conservation of fragmented communities. Chicago: University of Chicago, 1997. 616 p.

LAURANCE, W. F.; BIERREGAARD JUNIOR, R. O.; GASCON, C. Tropical forest fragmentation: synthesis of a diverse and dynamic discipline. Chicago: University of Chicago, 1997.

LOPES, L. E. Biologia comparada de Suiriri affinis e Suiriri islerorum (Aves: Tyrannidae) no cerrado do Brasil central. 2004. Dissertação (Mestrado) - Universidade de Brasília, Brasília, DF.

MACEDO, R. H. Reproductive patterns and social organization of the communal guira cuckoo (Guira guira) in central Brazil. Auk, [S.1.], v. 109, n. 4, p. 786-799, Oct./Dec. 1992. 
MANHÃES, M. A. Dieta de traupíneos (passeriformes, emberezidae) no parque estadual do ibitipoca, Minas Gerais, Brasil. Iheringia, Série Zoologia, Porto Alegre, v. 93, n. 1, p. 59-73, Mar. 2003.

MARCONDES-MACHADO, L. O. Comportamento alimentar de aves em Miconia rubiginosa (Melastomataceae) em fragmento de cerrado, São Paulo. Iheringia, Série Zoologia, [S.1.], v. 92, n. 3, p. 97-100, 2002. Disponível em: <http://www. scielo.br/pdf/isz/ v92n3/12981.pdf>. Acesso em: 1 ago. 2007.

MARINI, M. A. Foraging behavior and diet of the helmeted manakin. Condor, Santa Clara, v. 94, n. 1, p. 151-158, Jan./Feb. 1992.

MARINI, M. A.; AGUIAR, T. M.; ANDRADE, R. D.; LEITE, L. O.; ANCIÃES, M.; DUCA, C. G.; COELHO, M. M.; SEBAIO, F.; GONÇALVES, J. F. Biologia da nidificação de aves do sudeste de Minas Gerais. Revista Brasileira de Ornitologia, Ararajuba, 2006.

MARINI, M. A.; CAVALCANTI, R. B. Mating system of helmeted manakin (Antilphia galeata) in central Brazil. Auk, [S.1.], v. 109, n. 5, p. 911-913, Oct./Dec. 1992.

MARTINS, M. Ecologia dos cerrados de Itirapina. 2001. Disponível em: <http://eco. ib.usp.br/labvert/SiteItirapina/aves.htm $>$. Acesso em: 29 dez. 2007.

McGARIGAL, K.; MARKS, B. J. FRAGSTATS: spatial pattern analysis program for quantifying landscape structure. Washington, DC: USDA, 1995.

MENDONÇA, R. C.; FELFILI, J. M.; WALTER, B. M. T.; SILVA JÚNIOR, C.; REZENDE, A. V.; FILGUEIRAS, T. S.; NOGUEIRA, P. E. Flora vascular do cerrado. In: SANO, S.
M.; ALMEIDA. S. P. Cerrado, ambiente e flora. Planaltina: Embrapa-CPAC, 1998. p. 289-556.

MERCIVAL, F.; GALETTI, M. Aves como potenciais dispersoras de Ocotea pulchella Mart. (Lauraceae) numa área de vegetação de cerrado do sudeste brasileiro. Revista Brasileira de Botânica, São Paulo, v. 25, n. 1, p. 11-17, 2002. Disponível em: <http://www. scielo.br/pdf/rbb/v25n1/a03v25n1.pdf $>$. Acesso em: 1 ago. 2007.

MERRIAM, G. Are corridors necessary for the movement of biota? In: SAUNDERS, D. A.; HOBBS, R. J. (Eds.). Nature conservation 2: the role of corridors. Surrey Beattie and Sons: NSW, 1991. 442 p.

MERRIAM, G. Connectivity: a fundamental ecological characteristic of landscape pattern. In: INTERNATIONAL SEMINAR ON METHODOLOGY IN LANDSCAPE ECOLOGICAL RESEARCH AND PLANNING, 1., 1984, Denmark. Proceedings... Denmark: Roskilde University, 1984. p. 5-15.

METZGER, J. P. Estrutura da paisagem e fragmentação: análise bibliográfica. Anais da Academia Brasileira de Ciências, Rio de Janeiro, v. 71, n. 3, p. 445-463, 1999.

METZGER, J. P. Relationships between landscape structure and tree species diversity in tropical forests of South-East Brazil. Landscape and Urban Planning, Amsterdam, v. 37, n. 1, p. 29-35, June 1997.

METZGER, J. P. Tree functional group richness and landscape structure in a tropical fragmented landscape in SE Brazil. Ecological Applications, New York, v. 10, n. 4, p. 1147-1161, 2000. 
MOILANEN, A.; HANSKI, I. Metapopulation dynamics: effects of habitat quality and landscape structure. Ecology, Durham, v. 79, n. 7, p. 2503-2515, 1998.

MURCIA, C. Edge effects in fragmented forests: implications for conservation. Trends Ecology Evolution, Oxford, v. 10, n. 2, p. 58-62, Feb. 1995.

NOSS, R. F.; CSUTI, B.; GROOM, M. J. Habitat fragmentation: principles of conservation biology. Sunderland: Sinauer Association, 2006. 699 p.

NUNES, A. P.; TOM, W. M. Análise preliminar das relações biogeográficas da avifauna do Pantanal com biomas adjacentes. In: SIMPÓSIO SOBRE RECURSOS NATURAIS E SÓCIO-ECONÔMICOS DO PANTANAL, 4., 2004, Corumbá, MS. Anais... Corumbá: [s.n.], 2004. p. 1-8.

OCHOA-GAONA, S.; GONZALEZ-ESPINOSA, M.; MEAVE, J. A.; SORANI-DALBON, V. Effect of forest fragmentation on the woody flora of the highlands of Chiapas, Mexico. Biodiversity and Conservation, London, v. 13, n. 5, p. 867-884, May 2004.

RAGUSA-NETO, J. Raptors and "campo-cerrado" bird mixed flock led by Cypsnagra Hirundinacea (Emberizidae:Thraupinae). Revista Brasileira de Biologia, São Pau1o, v. 60, n. 3, p. 461-467, 2000. Disponível em: <http://www.scielo.br/pdf/ rbbio/ v60n3/3933.pdf>. Acesso em: 1 ago. 2007.

RATTER, J. A.; RIBEIRO, J. F.; BRIDGEWATER, S. The Brazilian Cerrado Vegetation and theats to its Biodiversity. Annal of Botany, London, v. 80, n. 3, p. 223-230, 1997.
RATTER, J. A.; RICHARDS, P. N.; ARGENTE, G. E.; GIFORD, D. R. G. Observations on the vegetation of northeastern Mato Grosso. Philosophical Transaction of the Royal Society of London, Series B.; Biological Sciences, London, v. 226, n. 880, p. 449-492, 1973.

RENJIFO, L. M. Effect of natural and anthropogenic landscape matrices on the abundance of subandean bird species. Ecological Applications, Tempe, v. 11, n. 1, p. 14-31, 2001.

RESTREPO, C.; GÓMEZ, N.; HEREDIA, S. Anthropogenic edges, treefall gaps, and fruit-frugivore interactions in a neotropical montane Forest Ecology, Amsterdam, v. 80, n. 2, p. 668-685, 1999.

RIBEIRO, J. F.; SILVA, J. C. S. Manutenção e recuperação da biodiversidade do Bioma Cerrado: o uso de plantas nativas. In: SIMPÓSIO SOBRE O CERRADO, 8., 1996, Brasília, DF. Anais... PEREIRA, R. C.; NASSER, L. C. B. Planaltina: Embrapa-CPAC, 1996. p. 10-14.

RIZZINI, C. T. Tratado de fitogeografia do Brasil. São Paulo: Hucitec/Edusp, 1979. v. 2, 347 p.

RODRIGUES, P. J. F. P.; NASCIMENTO, M. T. Fragmentação florestal: breves considerações teóricas sobre efeitos de borda. Rodriguésia, Rio de Janeiro, v. 57, n. 89, p. 63-74, 2006.

ROSENZWEIG, M. L. Species diversity in space and time. Cambridge: Cambridge University, 1995. $436 p$.

RUREMONDE, R. H. van; KALKHOVEN, J. T. Effects of woodlot isolation on the dispersion of plants with fleshy fruits. Journal of Vegetation Science, [S.1.], v. 2, n. 1, p. 377-384, June 1991. 
SANTOS, A. M. dos. Comunidades de aves em remanescentes florestais secundários de uma área rural no sudeste do Brasil. Revista Brasileira de Ornitologia, Ararajuba, v. 12, n. 1, p. 41-49, 2004.

SAUNDERS, D. A.; HOBBS, R. J. Nature conservation 2: the role of corridors. Chipping Norton: Surrey Beatty \& Sons Pty., 1991. $442 \mathrm{p}$.

SAUNDERS, D. A.; HOBBS, R. J.; MARGULES, C. R. Biological consequences of ecosystem fragmentation: a review. Conservation Biology, Cambridge, v. 5, n. 1, p. 1832, 1991.

SICK, H. Ornitologia brasileira. Rio de Janeiro: Nova Fronteira, 2001. 912 p.

SILVA, E. M. D. Composição e comportamento de grupos heteroespecíficos de aves em área de cerrado, no Distrito Federal. 1980. Dissertação (Mestrado) - Universidade de Brasília, Brasília, DF.

SILVA, J. M. C. Birds of the Cerrado Region, South America. Steenstrupia, Copenhagen, v. 21, n. 1, p. 69-92, 1995.

SILVA, J. M. C. da (Coord.). Ações prioritárias para a conservação da biodiversidade do cerrado e do Pantanal: aves. In: WORKSHOP "AÇÕES PRIORITÁRIAS PARA A CONSERVAÇÃO DA BIODIVERSIDADE DO CERRADO E PANTANAL", 1998. Anais... 1998a. Disponível em: <http://www.bdt.fat.org.br/workshop/ cerrado/br/aves $>$. Acesso em: 14 dez. 2007.
SILVA, J. M. C. da. Biogeografia e conservação de aves na região do cerrado e do Pantanal. In: WORKSHOP "AÇÕES PRIORITÁRIAS PARA A CONSERVAÇÃO DA BIODIVERSIDADE DO CERRADO E PANTANAL", 1998. Anais... 1998b. Disponível em: <http://www.bdt.fat.org.br/pdf/ workcerrado/AVES.PDF $>$. Acesso em: 14 dez. 2007.

SILVA, J. M. C. da. Endemic bird species and conservation in the Cerrado Region, South America. Biodiversity Conservation, London, v. 6, n. 1, p. 435-450, 1997.

SILVA, J. M. C.; BATES, J. M. Biogeographic patterns and conservation in South American cerrado: a tropical savanna hotspot. BioScience, [S.1.], v. 52, n. 1, p. 225-233, 2002.

SILVA, J. M. C.; UHL, C.; MURRAY, G. Plant succession, landscape management, and the ecology of frugivorous birds in abandoned Amazonian pastures. Conservation Biology, Cambridge, v. 10, n. 2, p. 491-503, Apr. 1996.

SILVA, W. R. de; MARCO, P.; HASUI, E.; GOMES, V. S. M. Patterns of fruit-frugivore interactions in two Atlantic forest bird communities of south-eastern Brazil: implications for conservation. In: LEVEY, D. J.; SILVA, W. R.; GALETTI, M. (Eds.). Seed dispersal and frugivory: ecology, evolution and conservation. Oxon: CABI, 2002. p. 423-435.

SILVEIRA, L. F.; D'HORTA, F. M. A avifauna da região de Vila Bela da Santíssima Trindade, Mato Grosso. Papéis Avulsos de Zoologia, São Paulo, v. 42, n. 10, p. 265-286. 2001. 
SIQUEIRA, P. R.; ROCHA, T. M. F.; TEIXEIRA, M. P.; LEITE, L. O.; BORGES, M. A. Z. A influência do tamanho corporal na dieta de nove espécies de aves da família Tyrandae. In: CONGRESSO DE ECOLOGIA DO BRASIL, 8., 2007, Caxambu. Anais... Caxambu: [s.n.], 2007.

SLATKIN, M. Gene flow and genetic drift in a species subject to frequent local extinctions. Theoretical Population Biology v. 12, p. 253-262. 1977.

SOULÉ, M. E.; GILPIN, M. E. Nature conservation 2: the role of corridors. [S.1.]: Surrey Beatty and Sons, 1991.

STOUFFER, P. C.; BIERREGAARD JUNIOR, R. O. Use of Amazonian forest fragments by understory insectivorous birds. Ecology, Durham, v. 76, n. 8 , p. 2429-2445, 1995 a.

STOUFFER, P. C.; BIERREGAARD JUNIOR, R. O. Effects of fragmentation on understory hummingbirds in Amazonian Brazil. Conservation Biology, Oxford, v. 9, n. 4, p. 1085-1094, 1995 b.

STRATFORD, J. A.; STOUFFER, P. C. Reduced feather growth rates of two common birds inhabiting central Amazonian forest fragments. Conservation Biology, Cambridge, v. 15, n. 3, p. 721-728, June 2001.

STRAUBE, F. C.; KRUL, R.; CARRANO, E. Coletânea da avifauna da região sul do estado do Paraná (Brasil): atualidades ornitológicas. 2005. Disponível em: <http://www.ao.com.br/ download/sulpr.pdf $>$. Acesso em: 15 jan. 1006.

THORNE, J. F. Landscape ecology. In: SMITH, D. S.; HELlMUND, P. C. (Eds.). Ecology of greenways. Minneapolis: [s.n.], 1993. p. 23-42.

TURNER, I. M.; CORLETT, R. T. The conservation value of small, isolated fragments of lowland tropical rain forest. Trends in Ecology and Evolution, [S.1.], v. 11, n. 8, p. 330-333, Aug. 1996.
UEZU, A. Composição e estrutura da comunidade de aves na paisagem fragmentada do Pontal do Paranapanema. 2006. 193 f. Tese (Doutorado) - Universidade de São Paulo, São Paulo.

UEZU, A. Efeito do tamanho do fragmento e do tipo de matriz na abundância de seis espécies de aves florestais em Caucaia do Alto, SP. 2002. 79 f. Dissertação (Mestrado em Ecologia) - Universidade de São Paulo, São Paulo.

UEZU, A.; BEYER, D. D.; METZGER, J. P. Can agroforest woodlots work as stepping stones for birds in the Atlantic Forest region? Biodiversity and Conservation, Oxford, 2008.

UEZU, A.; METZGER, J. P.; VIELLIARD, J. M. E. Effects on structural and functional connectivity and patch size on the abundance of seven Atlantic Forest bird species. Biological Conservation, Oxford, v. 123, n. 4, p. 507-519, 2005.

URBEN-FILHO, A.; GATTO, C. A. F. R.; STRAUBE, F. C. Avifauna do parque estadual do Cerrado, Jaguariaíva, Paraná. In: STRAUBE, F. C. et al. (Eds.). Ornitologia brasileira no Século XX. Curitiba: UNISUL; SOB, 2000. p. 347-348.

VALADÃO, R. M.; FRANCHIN, A. G.; MAGALHÃES JÚNIOR, O. A avifauna no Parque Municipal Victório Siquierolli, zona urbana de Uberlândia (MG). Biotemas, [S.1.], v. 19, n. 1, p. 81-91, mar. 2006.

VALERI, S. V.; SENÔ, M. A. A. F. A importância dos corredores ecológicos para a fauna e a sustentabilidade de remanescentes florestais. In: CONGRESSO INTERNACIONAL DE DIREITO AMBIENTAL, 8., 2004, São Paulo. Anais... São Paulo: [s.n.], 2004. p. 699-709.

VALÉRIO FILHO, M. Gerenciamento de bacias hidrográficas com aplicação de técnicas de geoprocessamento. In: TAUK-TORMELO, S. M. Análise ambiental: estratégias e ações. Rio Claro: Unesp, 1995. p. 135-140. 
VIANA, V. M. Biologia e manejo de fragmentos florestais naturais. In: CONGRESSO FLORESTAL BRASILEIRO, 6., 1990, Campos do Jordão, SP. Anais... Campos do Jordão: SBS/ SBEF, 1990. p. 113-118.

VRIJENHOEK, R.C. Gene flow and genetic diversity in naturally fragmented metapopulations of deep-sea hydrothermal vent animals. Journal of Heredity v. 88, n. 4, p. 285-293. 1997.
WICKAM, J. D.; RIITTERS, K. H.; WADE, T. G.; COULTON, J. W. Temporal change in forest fragmentation at multiple scales. Landscape Ecology, [S.1.], v. 22, n. 4, p. 481489, 2007.

WIENS, J. A.; SCHOOLEY, R. L.; WEEKS JUNIOR, R. D. Patchy landscapes and animal movements: do beetles percolate? Oikos, Copenhagen, v. 78, p. 257-264, 1997. 